\title{
Stress ulcer prophylaxis versus placebo-a blinded randomized control trial to evaluate the safety of two strategies in critically ill infants with congenital heart disease (SUPPRESS-CHD)
}

Kimberly I. Mills ${ }^{1,2+}$, Ben D. Albert ${ }^{2,3+}$, Lori J. Bechard ${ }^{2,3}$, Christopher P. Duggan ${ }^{2,4}$, Aditya Kaza ${ }^{2,5}$, Seth Rakoff-Nahoum ${ }^{2,6}$, Hera Vlamakis ${ }^{7}$, Lynn A. Sleeper ${ }^{1,2}$, Jane W. Newburger ${ }^{1,2}$, Gregory P. Priebe ${ }^{2,3}$ and Nilesh M. Mehta ${ }^{2,3,4^{*}}$ (iD)

\begin{abstract}
Background: Critically ill infants with congenital heart disease (CHD) are often prescribed stress ulcer prophylaxis (SUP) to prevent upper gastrointestinal bleeding, despite the low incidence of stress ulcers and limited data on the safety and efficacy of SUP in infants. Recently, SUP has been associated with an increased incidence of hospitalacquired infections, community-acquired pneumonia, and necrotizing enterocolitis. The objective of this pilot study is to investigate the feasibility of performing a randomized controlled trial to assess the safety and efficacy of withholding SUP in infants with congenital heart disease admitted to the cardiac intensive care unit.
\end{abstract}

Methods: A single center, prospective, double-blinded, randomized placebo-controlled pilot feasibility trial will be performed in infants with CHD admitted to the cardiac intensive care unit and anticipated to require respiratory support for $>24 \mathrm{~h}$. Patients will be randomized to receive a histamine-2 receptor antagonist (H2RA) or placebo until they are discontinued from respiratory support. Randomization will be performed within 2 strata defined by admission type (medical or surgical) and age (neonate, age $<30$ days, or infant, 1 month to 1 year). Allocation will be a 1:1 ratio using permuted blocks to ensure balanced allocations across the two treatment groups within each stratum. The primary outcomes include feasibility of screening, consent, timely allocation of study drug, and protocol adherence. The primary safety outcome is the rate of clinically significant upper gastrointestinal bleeding. The secondary outcomes are the difference in the relative and absolute abundance of the gut microbiota and functional microbial profiles between the two study groups. We plan to enroll 100 patients in this pilot study.

\footnotetext{
* Correspondence: nilesh.mehta@childrens.harvard.edu

${ }^{\dagger}$ Kimberly I. Mills and Ben D. Albert are the co-first authors.

${ }^{2}$ Harvard Medical School, Boston, MA, USA

${ }^{3}$ Division of Critical Care Medicine, Department of Anesthesiology, Critical

Care and Pain Medicine, Boston Children's Hospital, Boston, MA, USA

Full list of author information is available at the end of the article
}

(c) The Author(s). 2020 Open Access This article is licensed under a Creative Commons Attribution 4.0 International License, which permits use, sharing, adaptation, distribution and reproduction in any medium or format, as long as you give appropriate credit to the original author(s) and the source, provide a link to the Creative Commons licence, and indicate if changes were made. The images or other third party material in this article are included in the article's Creative Commons licence, unless indicated otherwise in a credit line to the material. If material is not included in the article's Creative Commons licence and your intended use is not permitted by statutory regulation or exceeds the permitted use, you will need to obtain permission directly from the copyright holder. To view a copy of this licence, visit http://creativecommons.org/licenses/by/4.0/ The Creative Commons Public Domain Dedication waiver (http://creativecommons.org/publicdomain/zero/1.0/) applies to the data made available in this article, unless otherwise stated in a credit line to the data. 
(Continued from previous page)

Discussion: Routine use of SUP to prevent upper gastrointestinal bleeding in infants is controversial due to a low incidence of bleeding events and concern for adverse effects. The role of SUP in infants with CHD has not been examined, and there is equipoise on the risks and benefits of withholding this therapy. In addition, this therapy has been discontinued in other neonatal populations due to the concern for hospital-acquired infections and necrotizing enterocolitis. Furthermore, exploring changes to the microbiome after exposure to SUP may highlight the mechanisms by which SUP impacts potential microbial dysbiosis of the gut and its association with hospitalacquired infections. Assessment of the feasibility of a trial of withholding SUP in critically ill infants with CHD will facilitate planning of a larger multicenter trial of safety and efficacy of SUP in this vulnerable population.

Trial registration: ClinicalTrials.gov, NCT03667703. Registered 12 September 2018, https://clinicaltrials.gov/ct2/ show/NCT03667703?term=SUPPRESS+CHD\&draw=2\&rank=1.

All WHO Trial Registration Data Set Criteria are met in this manuscript.

Keywords: Pediatric critical care, Pediatric cardiac critical care, Pediatric intensive care, H2 blocker, Congenital heart disease, Gastrointestinal hemorrhage, Infection, Microbiome

\section{Background}

Stress ulcer prophylaxis (SUP) is prescribed in the critically ill to decrease the incidence of stress-related mucosal damage that can lead to upper gastrointestinal (UGI) bleeding. The development of an UGI bleed while critically ill has been associated with increased intensive care unit (ICU) length of stay and mortality [1]. The adult practice of SUP administration has subsequently been adopted in neonatal and pediatric ICUs. However, the incidence of clinically significant UGI bleeds is very low, and there are limited data on the efficacy of SUP in critically ill infants and children [2]. Furthermore, the safety and efficacy of SUP has been called into question even in adults over the last decade [3-8].

In both adults and children, the incidence of clinically significant UGI bleeding is declining and hypothesized to be related to earlier initiation of enteral nutrition and increased utilization of goal-directed therapies [9]. Therefore, focus has shifted away from the utility of SUP and onto the safety and potential benefits of withholding this therapy. In critically ill adults, SUP has been associated with ventilator-associated pneumonia and Clostridioides difficile colitis [10, 11]. Similarly, in a large multicenter cohort, we reported a significant association between SUP and ventilator-associated pneumonia in critically ill children [8]. Several publications have also described an increase in bacteremia, necrotizing enterocolitis, and mortality in the neonatal ICU when patients were exposed to SUP $[3,5,6]$. These earlier studies have been limited by observational study design and potential confounding by indication. A definitive trial comparing administration versus withholding of SUP in critically ill infants with $\mathrm{CHD}$ is lacking. Based on the findings of prior studies, the role of SUP is being questioned $[4,12]$. Furthermore, recent advances in molecular methods have allowed a shift from traditional culture-based techniques to detect gut microbiota to high-throughput
DNA sequencing methods. The impact of acid suppression on the gut microbiota, especially the change in proportion of unfavorable organisms such as $C$. difficile and/or aerobic Gram-negative bacilli, may be an important mechanistic link between SUP and increased risk of infections.

Critically ill infants with congenital heart disease (CHD) are at risk for stress-related mucosal damage due to reduced splanchnic blood flow leading to mucosal ischemia and reperfusion injury, yet there is currently no consensus among pediatric cardiac intensivists regarding the clinical indications for SUP [13]. Therefore, the central objective of our study is to investigate the feasibility of conducting a clinical trial to assess the safety of withholding SUP in infants with CHD in the cardiac intensive care unit. Our hypothesis is that this trial design is feasible. We will also examine serial changes in the gut microbiota as a secondary aim to examine the difference in microbial profiles of patients receiving acid suppressive therapy compared to those receiving placebo. Our hypothesis is that patients receiving SUP will have decreased abundance and heterogeneity of gastrointestinal microbiota. Results of this feasibility trial will allow us to further refine inclusion and exclusion criteria, study procedures, data acquisition strategy, and study outcomes for a future multicenter, randomized controlled trial.

\section{Methods \\ Study design}

The study is a prospective, double-blinded randomized placebo-controlled pilot feasibility trial in a pediatric cardiac ICU at Boston Children's Hospital, a quaternary freestanding children's hospital. Enrollment began in February 2019 with a planned enrollment of 100 patients over a 2-year period. The local Institutional Review Board (IRB) at Boston Children's Hospital approved this study. All study protocol amendments, deviations, or 
adverse events will be immediately reported to IRB. All research team members, clinicians, data analysts, and trial participants are blinded to study assignments. Unblinding will only occur in the event a participant has a serious adverse event such a clinically significant gastrointestinal bleed. The study was registered at ClinicalTrials.gov (NCT03667703) and funded by The Gerber Foundation's National Research Grant (\#5781). The funding agency will have no access to or involvement in the data analysis or writing of the manuscripts. The research integrity, data quality, and adverse event assessment will be regularly reviewed by a pre-appointed, independent Data Safety Monitoring Board (DSMB). The DSMB has an appointed chair and five other members. Further details regarding the charter can be available by contacting the corresponding author who is a co-principal investigator. Table 1 shows the Standard Protocol Items: Recommendation for Interventional Trials (SPIRIT) schedule for the enrollment, intervention, and assessment periods. The SPIRIT checklist is in Additional file 1.

\section{Study population}

The inclusion and exclusion criteria are described in Table 2. Infants diagnosed with CHD admitted to the cardiac ICU and anticipated to require respiratory support (defined below) for greater than $24 \mathrm{~h}$ will be eligible for the study. Congenital heart disease includes anatomic, myopathic, and arrhythmic conditions. Respiratory support is defined as mechanical ventilation, including conventional, high frequency oscillatory, or jet ventilation, as well as non-invasive positive pressure ventilation, such as continuous (CPAP) and biphasic (BIPAP) positive airway pressure, and high-flow nasal cannula. Respiratory support for greater than $24 \mathrm{~h}$ was chosen as a surrogate for severity of illness. Patients will be excluded if they receive any form of antacid for $>7$ days during the past month as this could potentially alter their baseline gut microbiome. Patients will also be excluded if they are anticipated to receive high-dose steroids, intravenous non-steroidal anti-inflammatory agents, or high-dose aspirin during their hospitalization, as these medications may potentially cause gastritis and increase the risk for UGI bleeding. Finally, infants on certain anticoagulants-direct thrombin inhibitors and GPIIbIIIa inhibitors-will be excluded since these medications do not have an available reversal agent in the event of an UGI bleed.

\section{Study setting}

The study will be conducted in the cardiac intensive care unit at Boston Children's Hospital, a quaternary referral center and standalone children's hospital.

Table 1 Standard Protocol Items: Recommendation for Interventional Trials (SPIRIT) schedule of enrollment, interventions, and assessments

\begin{tabular}{|c|c|c|c|c|c|c|}
\hline \multirow[t]{3}{*}{ Time point } & & \multirow{3}{*}{$\begin{array}{l}\text { Enrollment } \\
\text { Pre-study }\end{array}$} & \multicolumn{4}{|l|}{ Study period } \\
\hline & & & \multicolumn{2}{|l|}{ Allocation } & \multirow{2}{*}{$\begin{array}{l}\text { Post-allocation } \\
24 \mathrm{~h} \text { after respiratory } \\
\text { support discontinued }\end{array}$} & \multirow{2}{*}{$\begin{array}{l}\text { Close-out } \\
\text { Hospital discharge }\end{array}$} \\
\hline & & & Study day \#1 & $\begin{array}{l}\text { Study day \#2 } \\
\text { through up to } 14\end{array}$ & & \\
\hline \multirow[t]{3}{*}{ Enrollment } & Eligibility screen & $\bullet$ & $\bullet$ & & & \\
\hline & Informed consent & $\bullet$ & $\bullet$ & & & \\
\hline & Allocation & & $\bullet$ & & & \\
\hline Intervention & $\mathrm{H} 2$ blocker or placebo & & $\bullet$ & $\bullet$ & & \\
\hline \multirow[t]{13}{*}{ Assessments } & Demographics & & $\bullet$ & $\bullet$ & $\bullet$ & \\
\hline & Laboratory data & & $\bullet$ & $\bullet$ & $\bullet$ & \\
\hline & Antibiotic exposure & & $\bullet$ & $\bullet$ & $\bullet$ & \\
\hline & Inotrope and vasoactive use & & $\bullet$ & $\bullet$ & $\bullet$ & \\
\hline & Nutritional support & & $\bullet$ & $\bullet$ & $\bullet$ & \\
\hline & Gastrointestinal bleeding & & $\bullet$ & $\bullet$ & $\bullet$ & $\bullet$ \\
\hline & Necrotizing enterocolitis & & $\bullet$ & $\bullet$ & $\bullet$ & $\bullet$ \\
\hline & Infectious complications & & $\bullet$ & $\bullet$ & $\bullet$ & $\bullet$ \\
\hline & Adverse event & & $\bullet$ & $\bullet$ & $\bullet$ & $\bullet$ \\
\hline & Oral, gastric, blood, and urine samples & & $\bullet$ & & $\bullet$ & $\bullet$ \\
\hline & Stool samples & & $\bullet$ & $\bullet$ & $\bullet$ & $\bullet$ \\
\hline & Length of stay & & & & & $\bullet$ \\
\hline & Mortality & & & & & $\bullet$ \\
\hline
\end{tabular}


Table 2 Eligibility criteria

Inclusion criteria
(1) $<12$ months of age (including premature newborns)
(3) Admitted to the CICU
(4) Anticipated to require respiratory support for $>24 \mathrm{~h}$ during their CICU stay
(5) Have received $\leq 1$ dose of stress ulcer prophylaxis ${ }^{\beta}$ during their current admission
(1) Prior use of antacids ${ }^{\beta}$ in the past month for $>7$ days
(2) Active gastrointestinal bleeding
(3) Active Helicobacter pylori infection
(4) Anticipated exposure to certain pharmaceuticals:
(i) High-dose steroids (equivalent to 4 mg/kg/day of methylprednisolone)
(ii) Intravenous non-steroidal anti-inflammatory drugs (i.e., ketorolac)
(iii) Certain anticoagulants including high-dose aspirin, direct thrombin inhibitors, and GPIIbllla inhibitors
(5) Planned to undergo or recently has undergone gastrointestinal surgery within the last 4 weeks
(6) Supported by ECMO or VAD
(7) Currently enrolled in another conflicting interventional trial
(8) Known to be allergic to H2RAs
(9) Admitted for palliative care
(10) Prior enrollment in the study
(11) Primary provider declines enrollment

CICU cardiac intensive care unit, H2RA histamine-2 receptor antagonist, PPI proton pump inhibitor, GllbIlla glycoprotein Ilb/Illa, ECMO extracorporeal membrane oxygenator, $V A D$ ventricular assist device

*CHD includes anatomic, myopathic, and arrhythmic conditions

"Respiratory support includes mechanical ventilation, non-invasive positive pressure ventilation, and high-flow oxygen therapy

${ }^{\beta}$ Stress ulcer prophylaxis or antacids include H2RAs, PPIs, and sucralfate

\section{Recruitment and study flow}

Potential patients will be screened for eligibility, and one of the principal investigators will approach the family of an eligible patient for written consent. If the legal guardian grants consent, the patient will be enrolled in the study and then randomized to one of two arms. Please see consent form in Supplementary files. Study procedures will be continued until the patient (1) no longer requires respiratory support for greater than $24 \mathrm{~h}$, (2) transfers to the floor or is discharged from the cardiac ICU, (3) completes 14 days of study drug, or (4) at any time during the study the primary provider believes that open-label acid suppression is indicated (Fig. 1). To ensure adequate enrollment and retention, we will provide education of the study objectives and procedures to important subspecialist groups, send mailers to eligible patients prior to delivery or surgery, and post signs about the study in the cardiac intensive care unit and preoperative clinic.

\section{Randomization}

Eligible patients will be randomly assigned by Boston Children's Research Pharmacy to receive either a histamine-2 receptor antagonist (i.e., ranitidine or famotidine per institutional standard) or placebo. The randomization assignments are generated by Boston Children's Hospital's proprietary randomization software, SciRan ${ }^{\oplus}$. Randomization will be performed within 2 strata defined by admission type (medical or surgical) and age (neonate, age $<30$ days, or infant, 1 month to 1 year). Allocation will be using permuted blocks in a 1:1 ratio to ensure balanced allocations across the two treatment groups within each stratum. Allocation concealment is achieved by ensuring that only the pharmacy team holds the randomization key. The pharmacy does not partake in the outcome variable assessment, and the study investigators do not have access to the randomization key.

\section{Sample size}

We powered the precision of our feasibility estimates for both screening and drug initiation-which we deemed as the two most important feasibility measures. There are approximately 600 patients under 1 year of age admitted to the cardiac ICU each year, and we anticipate that an estimated 200 patients will be eligible over the 2-year recruitment period. The target to demonstrate feasibility is screening $80 \%$ of all patients. If $n=600$, the lower limit of the $95 \%$ one-sided confidence interval will include $80 \%$ as long as the observed screening rate is at least $82.7 \%$. There are 100 patients to be randomized in this pilot trial. The target to demonstrate feasibility with respect to drug initiation is to have $80 \%$ of randomized patients receive their first dose of study drug within $48 \mathrm{~h}$. With $n=100$, the lower limit of the $95 \%$ one-sided confidence interval will include $80 \%$ as long as the observed drug initiation rate is at least $86.9 \%$. That is, as long as the observed drug initiation rate is at least $86.9 \%$, we can be $95 \%$ confident that the drug initiation rate, to be realized in a future trial, is at least $80 \%$. The study is not powered to assess a statistical difference between the incidence of UGI bleeding and hospital-acquired infections, as the historical incidences are very low, $0.5 \%$ and $2 \%$, respectively. These outcomes will be further assessed in a future larger, multicenter trial.

For the assessment of oral, gastric, and stool microbiota, a total of 600 samples (2 samples per site) will be 


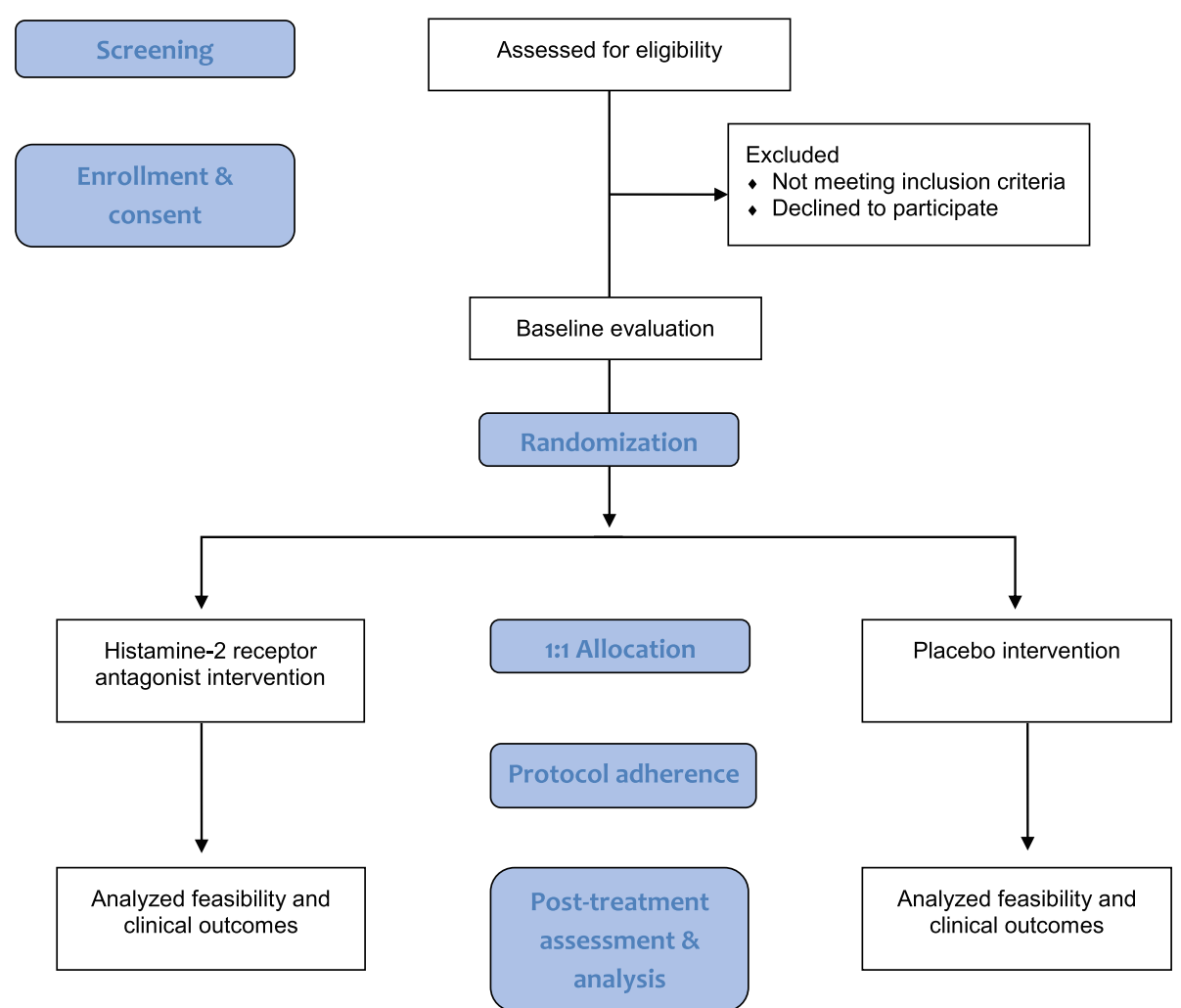

Fig. 1 Study flowchart of trial design

obtained from the 100 patients. We estimate detectable effect sizes using these sample sizes based on the $\mathrm{Hu}$ man Microbiome Project [14]. With adjustment for three covariates: one categorical binary (antibiotics), one continuous (age), and one categorical tertiary (hospitalacquired infections), we anticipate a power of 0.9 to detect a $1.36 \%$ level of rare taxon relative abundance and a $3.46 \%$ level of common taxa relative abundance, within a treatment arm between two time points, and similarly between two treatment arms at a given time point for either oral, gastric, or stool samples. All estimates incorporate stringent Bonferroni multiple-hypothesis correction to an adjusted $p$ value of 0.05 . Approximating as above, the corresponding detection limit for 250 metagenomic pathways with power of 0.9 would be $3.87 \%$.

\section{Study intervention}

Study participants will be randomly assigned to receive a histamine- 2 receptor antagonist (i.e., ranitidine or famotidine) or placebo. As there are no pediatric-specific recommendations regarding type of SUP, we elected to conduct our study with a histamine-2 receptor antagonist since this is the current standard practice at our institution. In this pragmatic design, participants can receive study drug either intravenously or enterally, depending on the clinician's preference. The dosing is based on age and route. For ranitidine, neonates $(<31$ days old) will receive either $1 \mathrm{mg} / \mathrm{kg}$ intravenously/enterally every $12 \mathrm{~h}$, and infants ( $\geq 31$ days old) will receive $1 \mathrm{mg} / \mathrm{kg}$ intravenously/enterally every $8 \mathrm{~h}$. For famotidine, if the participant is $<3$ months, they will receive $0.5 \mathrm{mg} / \mathrm{kg} /$ dose IV/ PO daily, and if $>3$ months old, they will receive 0.25 $\mathrm{mg} / \mathrm{kg} /$ dose IV every $12 \mathrm{~h}$ or $0.5 \mathrm{mg} / \mathrm{kg} /$ dose PO every $12 \mathrm{~h}$. As part of pharmacovigilance, surveillance for hepatic dysfunction and thrombocytopenia will be included in the case report forms.

The placebo will be in an equivalent volume of $0.9 \%$ saline intravenously or a stevioside sugar-free syrup vehicle that resembles the color, tonicity, and texture of oral ranitidine or famotidine. Individualized unit-dose syringes will be provided to each study participant and blinded to the study team, bedside clinicians, parents, and outcome assessors. All interventions other than the study drug are left to the discretion of the treating clinicians. Once a participant completes the study, the clinicians can prescribe acid suppression per their usual practice. Adherence to intervention protocols is monitored daily by research staff by checking in with participant's bedside nurse to ensure medication administration and timely sample collection. The research staff also speaks with the attending physician daily to assess for any adverse events or study-related issues. Patients with 
hemodynamically significant UGI bleeding will be withdrawn from the study, as they would likely require openlabel acid suppression. There also exist study-halting criteria, which would stop the study until the DSMB reviewed and recommended continuation of the study. Final determination of trial termination will be made by principal investigators, if necessary. Auditing of trial conduct is done every 6 months by the DSMB, independent of the investigators and sponsor. The study-halting criteria include a total of 3 UGI bleeding events or an enrollment number of less than 20 patients per year. The principal investigators will have access to the final trial dataset.

\section{Measurements and definitions}

Data will be imported into a secure, password-protected, FDA-compliant database (InForm ${ }^{\circ}$ Electronic Data Capture). Data collection will include demographic, procedural, laboratory, pharmaceutical, nutritional, ventilatory, and outcome variables. Samples obtained will include oral swabs, gastric aspirates (via indwelling NG tube), discarded blood, and urine at the initiation and conclusion of the study. In addition, serial stool samples will be collected while on study. Data are collected in daily case report forms (CRFs) and then imported weekly into the InForm ITM (Integrated Trial Management) System. Monthly audits of the InForm database are completed with the study investigators to ensure completeness and minimize transcription errors. In addition, the InForm database has safety metrics built in for out of bounds values.

Important definitions include the following:

(1) Clinically significant UGI bleed-new-onset bleeding from the UGI tract (i.e., hematemesis, bloody gastric aspirate, or hematochezia) AND associated with (a) decrease in hemoglobin by $2 \mathrm{~g} /$ $\mathrm{dL}$, OR (b) decline in mean arterial blood pressure by $10 \mathrm{mmHg}$ or initiation/increase of inotrope/ vasoactive medications, OR (c) increase in heart rate by 20 beats per minute in the absence of an arrhythmia or fever, OR (d) need for unanticipated blood transfusion, OR (e) unexpected endoscopic or operative procedure to achieve hemostasis. This definition has been used in adult randomized controlled trials with excellent inter-rater agreement $[15,16]$.

(2) Bloodstream infection (BSI) - a laboratoryconfirmed bloodstream infection with or without a central line in place.

(3) Ventilator-associated event (VAE) - a deterioration in respiratory status after a period of stability or improvement on the ventilator, evidence of infection or inflammation and laboratory evidence of a respiratory infection (CDC).
(4) Urinary tract infection-a laboratory-confirmed urinary tract infection with or without a urinary catheter in place.

(5) Clostridium difficile associated diarrhea-diarrhea in the presence of a positive $C$. difficile test.

(6) Mediastinitis-a laboratory-confirmed organism from mediastinal tissue or fluid, or based on gross anatomic exam, or has hyper/hypothermia or apnea or bradycardia or sternal instability with at least one of the following: (a) purulent drainage from the mediastinal area or (b) mediastinal widening on imaging (CDC).

(7) Superficial wound infection-has two of the following symptoms: (a) erythema, (b) tenderness, (c) swelling AND a laboratory-confirmed organism is identified from the wound.

(8) Gastrointestinal microbiota-difference in oral and stool microbiome between the 2 groups in this study will be examined.

\section{Sample analyses}

For assessment of stool microbiota, sequence-based microbial community surveys of stool samples will be carried out by $16 \mathrm{~S}$ rRNA gene-based sequencing in the Microbial Genomics and Transcriptomics Core at the Broad Institute. Their protocol targets the V4 window of the $16 \mathrm{~S}$ rRNA gene and uses Illumina MiSeq system at the Broad Genomics Platform to produce on average 25, 000 quality filtered, stitched paired-end reads per sample, representing the current state-of-the-art [17]. In addition, a higher resolution community survey and complementary functional survey of a subset of the stool samples (20\%) will be obtained using metagenomic shotgun DNA sequencing, to be performed at the Broad Institute in the Broad Technology Labs and Genomics Platform. Metagenomic libraries will be constructed using the Nextera XT DNA library preparation kit (Illumina) and sequencing will be performed on an Illumina HiSeq 2000 to generate a minimum of $2 \mathrm{~Gb}$ of $101 \mathrm{nt}$ paired-end reads.

For evaluation of oral and gastric microbiota, samples will be subjected to ribosomal DNA (rDNA) amplicon sequencing to characterize the composition of these microbiome communities. First, DNA will be extracted using a robust commercial extraction kit. Next, rDNA hypervariable regions of specific kingdoms will be amplified using universal primers. For bacteria, 16S V3V4 will be targeted using 5 '-CCTACGGGNGGCWGCAG-3' and 5'-GGACTACNVGGGTWTCTAAT-3'; for fungi, ITS1 is targeted using $5^{\prime}$-CTYGGTCATTTAGAGGAA GTAA-3' and 5'-GCTGCGTTCTTCATCGATGC-3'. Specifically, Illumina adapter sequences and variable spacers are added $5^{\prime}$ upstream of these sequences to enable high-throughput multiplexed sequencing. The 
prepared amplicons will then be pooled and sequenced using Illumina Miseq $2 \times 300$ bp platform. Illumina raw reads will be de-multiplexed, quality trimmed, dereplicated and denoised, and finally mapped against established rDNA databases. The derived OTU table will document the relative abundance of each taxonomy within each sample.

\section{Outcomes}

The central objective for our pilot study is to investigate whether a clinical trial assessing the safety and efficacy of withholding SUP in infants with CHD in the cardiac ICU is feasible. The trial will be considered feasible if each of the following 4 a priori variables are met: (1) > $80 \%$ of eligible patients are approached for consent (screening), (2) $>20 \%$ of eligible patients are randomized (enrollment and consent), (3) $>80 \%$ of consented patients received their first dose of study drug within $48 \mathrm{~h}$ (allocation), and (4) $>80 \%$ protocol compliance achieved (protocol adherence). Adherence to the protocol is defined as having received all doses of study drug as prescribed during the study period. Protocol deviation is defined as either premature termination of the study or prescription of SUP that is not part of the study while enrolled. In addition to feasibility, we will assess safety by comparing the difference in the incidence of clinically significant UGI bleeding and hospital-acquired infections between participants receiving SUP versus placebo. Finally, we will explore the changes to the gut microbiota by comparing the absolute and serial differences in the abundance of bacteria and functional microbial profiles between those receiving SUP compared to placebo. The study investigators will not be blinded to primary outcome measures. The microbiome specimens (for secondary outcome measure) will be processed and analyzed without revealing their study group allocation and thus will be blinded.

\section{Statistics}

The study will be double-blinded, and we will utilize an intention-to-treat (primary analysis) and per protocol (secondary) analysis. Trial participants who do not complete the intervention will remain in the primary, intention-to-treat analysis of trial outcomes. Data will be reviewed every 6 months by the DSMB. Homogeneity of the two treatment arms will be assessed using a Fisher exact or chi-square test for categorical variables and a Student $t$ test (parametric) or Wilcoxon rank sum test (non-parametric) for continuous variables.

\section{Feasibility analysis}

For each feasibility outcome measure, we will report the proportions of screened patients and/or participants meeting each criterion successfully and the associated one-sided 95\% confidence interval.

\section{Safety analysis}

A two-sided 95\% confidence interval will be constructed for treatment difference in the proportion of patients with UGI bleeding and hospital-acquired infections, as well as for each treatment-arm specific rate of UGI bleeding.

\section{Microbiome analysis}

Once the rRNA sequencing is completed, we will perform taxonomic profiling to identify distinct microbial lineages and then compare them to the published Greengenes, SILVA (for 16S), and UNITE (for ITS1) Reference Database [18-20]. The primary characteristics to be assessed between treatment arms are as follows: (a) within-sample and between-sample overall ecology of the microbial community, (b) absolute and relative abundance of microbial communities, and (c) pattern classification analysis to identify diversity [21]. We will then perform per-feature multivariable association analyses that estimate which microbiome attributes differ by treatment arm as well as between time points, while accounting for covariates, to identify how microbes are affected by outcomes in the presence of certain covariates [22-24]. We will adjust for delivery type (C-section vs. vaginal delivery), nutrition type (breast milk vs. formula), and antibiotics prescribed to the patient (not mother) as these are known confounders in the infant gut microbiome.

\section{Discussion}

The practice of routine acid suppressive therapy in infants admitted to the CICU must be examined. Rising concerns related to the adverse effects of acid suppression, particularly hospital-acquired infections, must be acknowledged and the indications for this therapy need to be revisited. Therefore, we have proposed a pilot feasibility trial to explore the safety of withholding SUP and examine the changes to the microbiome after exposure to SUP in this population. Our trial will highlight the mechanisms by which SUP impacts the microbial dysbiosis of the gut and its association with hospitalacquired infections. If withholding of SUP is deemed safe and associated with less disruption of the gut microbial profile, it could guide a significant change in practice in centers where this therapy is routinely prescribed.

Although considered the gold standard, there are several barriers to conducting high-quality randomized controlled trials in pediatric critical care. Included in these barriers are scarcity of research funding and failure to complete the trial due to difficulty with patient enrollment in the ICU environment [12]. Given these hurdles, large randomized controlled trials are sparse in pediatric critical care and several trials have been stopped early, 
most commonly due to futility $[25,26]$. To address the challenges with timely completion of a trial, certain governing bodies explicitly recommend that feasibility and pilot studies be conducted prior to undertaking and funding larger randomized controlled trials [27]. At the conclusion of our feasibility trial, we will be able to identify important barriers and, if necessary, amend the study protocol in order to proceed with a larger randomized controlled trial in the future. If this pilot trial concludes that a larger trial is feasible, we plan to conduct a noninferiority, multicenter randomized controlled trial in pediatric cardiac ICUs focusing on clinical outcomes shortly thereafter. We hypothesize that withholding SUP in critically ill infants with CHD does not increase the incidence of UGI bleeding and results in less disruption of the gut microbiota, possibly decreasing the incidence of hospital-acquired infections, compared to SUP therapy. The results of our study will potentially define appropriate indications for SUP in infants admitted to the CICU and illuminate the mechanistic relationship between microbial alterations and outcomes during critical illness.

There are a few limitations we have identified in our current study protocol. First, the anticipated duration of respiratory support of more than $24 \mathrm{~h}$ was chosen as an enrollment criterion because it will exclude patients with short ICU stays and exposure to the intervention. To examine whether anticipated duration of respiratory support is a reasonable marker for length of stay and exposure to the intervention, we plan to compare actual duration of respiratory support and length of stay in those who screened in versus those who screened out. Second, obtaining gastric samples may be difficult in participants as infants often make very little gastric fluid when critically ill. Finally, some infants might not have regular bowel movements, limiting the frequency of stool samples and thereby limiting the longitudinal or treatment effect assessment.

\section{Trial status}

The submitted protocol is the 3rd version, last amended on October 17, 2019. Recruitment for the study began on February 4, 2019, and should be completed by January 2021. The recent recall of ranitidine prompted removal of that drug from the institutional formulary, and famotidine will be the histamine- 2 antagonist for this study.

\section{Supplementary information}

Supplementary information accompanies this paper at https://doi.org/10. 1186/s13063-020-04513-w.

Additional file 1. SPIRIT 2013 Checklist: Recommended items to address in a clinical trial protocol and related documents.

\section{Abbreviations}

H2RA: Histamine-2 receptor antagonist; UGI: Upper gastrointestinal; SUP: Stress ulcer prophylaxis; ICU: Intensive care unit; CHD: Congenital heart disease; IRB: Institutional Review Board; DSMB: Data Safety Monitoring Board; SPIRIT: Standard Protocol Items: Recommendation for Interventional Trials; CPAP: Continuous positive airway pressure; BIPAP: Biphasic positive airway pressure

\section{Acknowledgements}

The study was funded by The Gerber Foundation. We acknowledge the Pediatric Anesthesia Research Center (PARC), funded by the Department of Anesthesiology, Critical Care and Pain Medicine at Boston Children's Hospital, for providing a research nurse, Brooke Sens, RN, and assistant, Sheng Huang. We would also like to acknowledge the funding support of the Harvard Catalyst Clinical Research Center and the Institutional Centers for Clinical and Translational Research (ICCTR) at Boston Children's Hospital. CD was supported in part by K24 DK104676 and P30 DK040561.

\section{Authors' contributions}

Each of the authors (KIM, BDA, LJB, GPP, CD, LAS, AK, JN, and NMM) made substantial contributions to the concept and design of the work. KIM, BDA, and NMM drafted the manuscript, and all of the authors (KIM, BDA, LJB, GPP, $C D, L A S, A K, J N$, and NMM) edited and approved the submitted version of the manuscript. Each author (KIM, BDA, LJB, GPP, CD, LAS, AK, JN, and NMM) is accountable for their contributions to the work. No professional writers will be used for the manuscript.

\section{Funding}

The study is funded by The Gerber Foundation's National Research Grant (Project ID: 5781). The Gerber Foundation will not be involved in the design, execution, or analysis of the trial and will not participate in the drafting of the manuscript. Contact information: tgf@gerberfoundation.org; phone (231) 924-3175.

\section{Availability of data and materials}

The results of the trial are the whole property of the investigators and the institution and will be published in scientific journals without restriction. The datasets used and/or analyzed during the current study are available from the corresponding author on reasonable request.

\section{Ethics approval and consent to participate}

The Institutional Review Board at Boston Children's Hospital approved the study (IRB-A00028715-1). Information regarding the study will be presented to the parents in written and informed consent. Written informed consent will be obtained from at least one legal guardian of the participant, and the right to withdraw from the study at any time will be emphasized.

\section{Consent for publication}

Not applicable

\section{Competing interests}

The authors declare that they have no competing interests.

\section{Author details}

${ }^{1}$ Department of Cardiology, Boston Children's Hospital, Boston, MA, USA. ${ }^{2}$ Harvard Medical School, Boston, MA, USA. ${ }^{3}$ Division of Critical Care Medicine, Department of Anesthesiology, Critical Care and Pain Medicine, Boston Children's Hospital, Boston, MA, USA. ${ }^{4}$ Center for Nutrition, Boston Children's Hospital, Boston, MA, USA. ${ }^{5}$ Department of Cardiac Surgery, Boston Children's Hospital, Boston, MA, USA. ${ }^{6}$ Department of Pediatrics, Boston Children's Hospital, Boston, MA, USA. ${ }^{7}$ Broad Institute of MIT and Harvard, Boston, MA, USA.

Received: 27 December 2019 Accepted: 15 June 2020

Published online: 29 June 2020

\section{References}

1. Cook DJ, Griffith LE, Walter SD, Guyatt GH, Meade MO, Heyland DK, et al. The attributable mortality and length of intensive care unit stay of clinically important gastrointestinal bleeding in critically ill patients. Crit Care (London, England). 2001;5(6):368-75. 
2. Deerojanawong J, Peongsujarit D, Vivatvakin B, Prapphal N. Incidence and risk factors of upper gastrointestinal bleeding in mechanically ventilated children. Pediatr Crit Care Med. 2009;10(1):91-5.

3. Terrin G, Passariello A, De Curtis M, Manguso F, Salvia G, Lega L, et al. Ranitidine is associated with infections, necrotizing enterocolitis, and fatal outcome in newborns. Pediatrics. 2012;129(1):e40-5.

4. Selvanderan SP, Summers MJ, Finnis ME, Plummer MP, Ali Abdelhamid Y, Anderson MB, et al. Pantoprazole or placebo for stress ulcer prophylaxis (POP-UP): randomized double-blind exploratory study. Crit Care Med. 2016; 44(10):1842-50.

5. Guillet R, Stoll BJ, Cotten CM, Gantz M, McDonald S, Poole WK, et al. Association of $\mathrm{H}$ 2-blocker therapy and higher incidence of necrotizing enterocolitis in very low birth weight infants. Pediatrics. 2006;117(2):e13742.

6. Bianconi S, Gudavalli M, Sutija VG, Lopez AL, Barillas-Arias L, Ron N. Ranitidine and late-onset sepsis in the neonatal intensive care unit. J Perinat Med. 2007:35(2):147-50.

7. Alhazzani W, Alshamsi F, Belley-Cote E, Heels-Ansdell D, BrignardelloPetersen R, Alquraini M, et al. Efficacy and safety of stress ulcer prophylaxis in critically ill patients: a network meta-analysis of randomized trials. Intensive Care Med. 2018;44(1):1-11.

8. Albert BD, Zurakowski D, Bechard LJ, Priebe GP, Duggan CP, Heyland DK, et al. Enteral nutrition and acid-suppressive therapy in the PICU: impact on the risk of ventilator-associated pneumonia. Pediatr Crit Care Med. 2016; 17(10):924-9.

9. Faisy C, Guerot E, Diehl JL, Iftimovici E, Fagon JY. Clinically significant gastrointestinal bleeding in critically ill patients with and without stressulcer prophylaxis. Intensive Care Med. 2003;29(8):1306-13.

10. Yearsley KA, Gilby LJ, Ramadas AV, Kubiak EM, Fone DL, Allison MC. Proton pump inhibitor therapy is a risk factor for Clostridium difficile-associated diarrhoea. Aliment Pharmacol Ther. 2006;24(4):613-9.

11. Cook DJ, Reeve BK, Guyatt GH, Heyland DK, Griffith LE, Buckingham L, et al. Stress ulcer prophylaxis in critically ill patients. Resolving discordant metaanalyses. JAMA. 1996;275(4):308-14.

12. Duffett M, Choong K, Foster J, Gilfoyle E, Lacroix J, Pai N, et al. Pediatric intensive care stress ulcer prevention (PIC-UP): a protocol for a pilot randomized trial. Pilot Feasibility Stud. 2017;3:26.

13. Costarino AT, Dai D, Feng R, Feudtner C, Guevara JP. Gastric acid suppressant prophylaxis in pediatric intensive care: current practice as reflected in a large administrative database. Pediatr Crit Care Med. 2015; 16(7):605-12.

14. Human Microbiome Project Consortium. A framework for human microbiome research. Nature. 2012;486(7402):215-21.

15. Arnold DM, Donahoe L, Clarke FJ, Tkaczyk AJ, Heels-Ansdell D, Zytaruk N, et al. Bleeding during critical illness: a prospective cohort study using a new measurement tool. Clin Invest Med. 2007;30(2):E93-102.

16. Arnold DM, Lauzier F, Rabbat C, Zytaruk N, Barlow Cash B, Clarke F, et al. Adjudication of bleeding outcomes in an international thromboprophylaxis trial in critical illness. Thromb Res. 2013;131(3):204-9.

17. Caporaso JG, Lauber CL, Walters WA, Berg-Lyons D, Huntley J, Fierer N, et al. Ultra-high-throughput microbial community analysis on the Illumina HiSeq and MiSeq platforms. ISME J. 2012;6(8):1621-4.

18. Callahan BJ, McMurdie PJ, Rosen MJ, Han AW, Johnson AJ, Holmes SP. DADA2: high-resolution sample inference from Illumina amplicon data. Nat Methods. 2016;13(7):581-3.

19. McDonald D, Price MN, Goodrich J, Nawrocki EP, DeSantis TZ, Probst A, et al. An improved Greengenes taxonomy with explicit ranks for ecologica and evolutionary analyses of bacteria and archaea. ISME J. 2012;6(3):610-8.

20. Wang Q, Garrity GM, Tiedje JM, Cole JR. Naive Bayesian classifier for rapid assignment of rRNA sequences into the new bacterial taxonomy. Appl Environ Microbiol. 2007:73(16):5261-7.

21. McMurdie PJ, Holmes S. phyloseq: an R package for reproducible interactive analysis and graphics of microbiome census data. PLoS One. 2013;8(4): e61217.

22. Mallick H, Ma S, Franzosa EA, Vatanen T, Morgan XC, Huttenhower C. Experimental design and quantitative analysis of microbial community multiomics. Genome Biol. 2017;18(1):228.

23. Smyth GK. Linear models and empirical bayes methods for assessing differential expression in microarray experiments. Stat Appl Genet Mol Biol. 2004;3:Article3.
24. Trapnell C, Hendrickson DG, Sauvageau M, Goff L, Rinn JL, Pachter L. Differential analysis of gene regulation at transcript resolution with RNA-seq Nat Biotechnol. 2013;31(1):46-53.

25. Choong K, Duffett M, Cook DJ, Randolph AG. The impact of clinical trials conducted by research networks in pediatric critical care. Pediatr Crit Care Med. 2016;17(9):837-44.

26. Duffett M, Choong K, Hartling L, Menon K, Thabane L, Cook DJ. Randomized controlled trials in pediatric critical care: a scoping review. Crit Care (London, England). 2013;17(5):R256.

27. Craig P, Dieppe P, Macintyre S, Michie S, Nazareth I, Petticrew M. Developing and evaluating complex interventions: the new Medical Research Council guidance. BMJ (Clinical research ed). 2008;337:a1655.

\section{Publisher's Note}

Springer Nature remains neutral with regard to jurisdictional claims in published maps and institutional affiliations.
Ready to submit your research? Choose BMC and benefit from:

- fast, convenient online submission

- thorough peer review by experienced researchers in your field

- rapid publication on acceptance

- support for research data, including large and complex data types

- gold Open Access which fosters wider collaboration and increased citations

- maximum visibility for your research: over $100 \mathrm{M}$ website views per year

At BMC, research is always in progress.

Learn more biomedcentral.com/submissions 